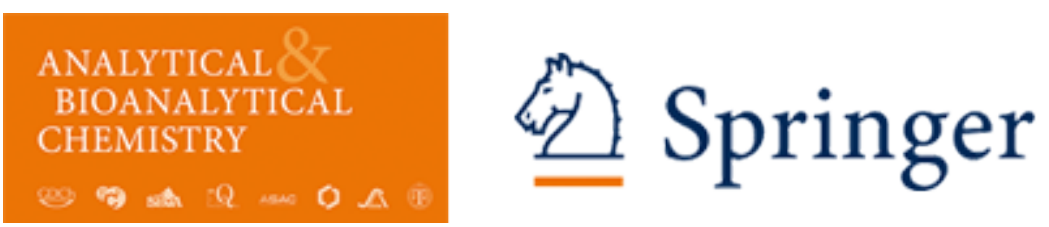

\title{
Programmable RNA-based Systems for Sensing and Diagnostic Applications
}

\begin{tabular}{|r|l|}
\hline Journal: & Analytical and Bioanalytical Chemistry \\
\hline Manuscript ID & Draft \\
\hline Type of Paper: & Trends \\
\hline Autho Submitted by the \\
Complete List of Authors: & n/a \\
& $\begin{array}{l}\text { rossetti, marianna; Universita degli Studi di Roma Tor Vergata } \\
\text { Del Grosso, Erica; Universita degli Studi di Roma Tor Vergata } \\
\text { Ranallo, Simona; Universita degli Studi di Roma Tor Vergata } \\
\text { mariottini, davide; Universita degli Studi di Roma Tor Vergata } \\
\text { Idili, Andrea; University of California Santa Barbara, Chemistry and } \\
\text { Biochemistry; University of California Santa Barbara Institute for } \\
\text { Collaborative Biotechnologies, Center for Bioengineering } \\
\text { Bertucci, Alessandro; University of California San Diego CNRS Joint } \\
\text { Chemistry Laboratory; Universita degli Studi di Roma Tor Vergata } \\
\text { Porchetta, Alessandro; Universitá Roma, Dipartimento di Scienze e } \\
\text { Tecnologie Chimiche }\end{array}$ \\
\hline Keywords: & RNA aptamers, diagnostics, toehold switches, RNA nanotechnology. \\
\hline & \\
\hline
\end{tabular}




\title{
Programmable RNA-based systems for sensing and diagnostic applications
}

Marianna Rossetti, ${ }^{1}$ Erica Del Grosso, ${ }^{1}$ Simona Ranallo, ${ }^{1}$ Davide Mariottini, ${ }^{1}$ Andrea Idili, ${ }^{2}$ Alessandro Bertucci, ${ }^{1,3 *}$ Alessandro Porchetta ${ }^{1 *}$

${ }^{1}$ Department of Chemical Sciences and Technologies, University of Rome Tor Vergata, Rome, 00133, Italy

${ }^{2}$ Department of Chemistry and Biochemistry, University of California Santa Barbara, CA 93106, USA

${ }^{3}$ Department of Chemistry and Biochemistry, University of California San Diego, La Jolla, CA 92093, USA

* Alessandro Bertucci

alessandro.bertucci@uniroma2.it

* Alessandro Porchetta

alessandro.porchetta@uniroma2.it

\begin{abstract}
The emerging field of RNA nanotechnology harnesses the versatility of RNA molecules to generate nature-inspired systems with programmable structure and functionality. Such methodology has therefore gained appeal in the fields of biosensing and diagnostics, where specific molecular recognition and advanced input/output processing are demanded. The use of RNA modules and components allows for achieving diversity in structure and function, for processing information with molecular precision, and for programming dynamic operations on the grounds of predictable non-covalent interactions. When RNA nanotechnology meets bioanalytical chemistry, sensing of target molecules can be performed by harnessing programmable interactions of RNA modules, advanced field-ready biosensors can be manufactured by interfacing RNA-based devices with supporting portable platforms, and RNA sensors can be engineered to be genetically encoded allowing for real-time imaging of biomolecules in living cells. In this article, we report recent advances in RNA-based sensing technologies and discuss current trends in RNA nanotechnologyenabled biomedical diagnostics. In particular, we describe programmable sensors that leverage modular designs comprising dynamic aptamer-based units, synthetic RNA nanodevices able to
\end{abstract}


perform target-responsive regulation of gene expression, and paper-based sensors incorporating artificial RNA networks.

\section{Graphical abstract}

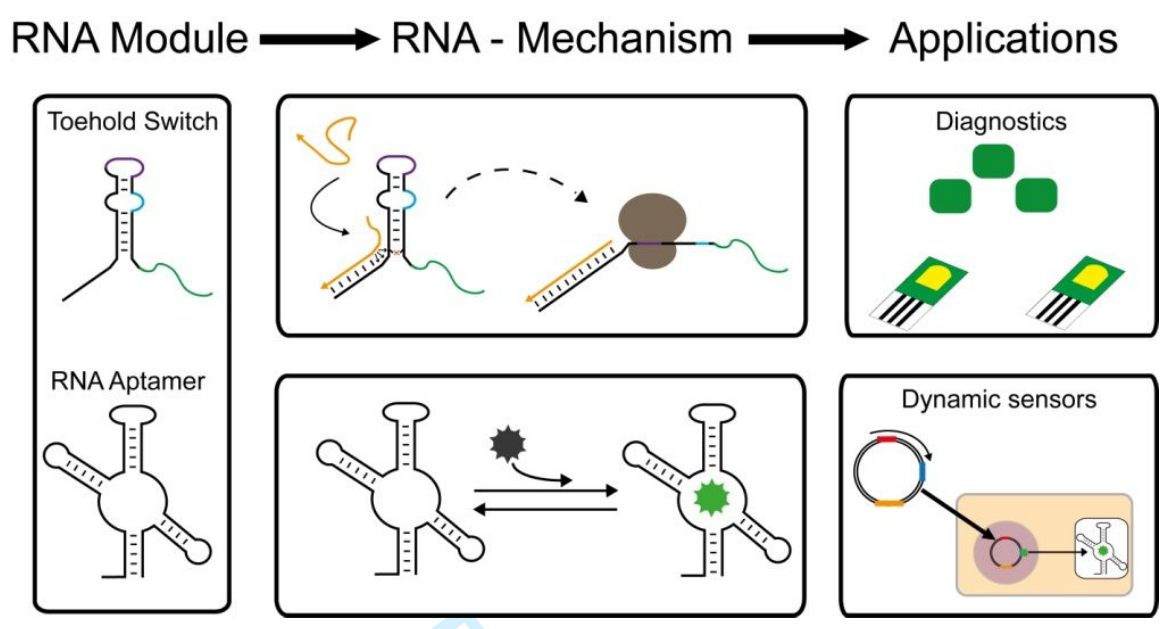

\section{Keywords}

RNA aptamers, diagnostics, synthetic biology, toehold switches, RNA nanotechnology. 


\section{Introduction}

RNA is an extraordinary versatile molecule with its structure and functionality determined by the Watson-Crick base pairing rules. This allows for intra- and intermolecular interactions to be readily predicted on the grounds of a simple set of defined non-covalent bonds. Non-canonical base pairing (i.e. Hoogsteen interactions), the many available tertiary structures (i.e. G-quadruplex motifs) [1], and the affinity for diverse ligands such as proteins and small molecules all contribute to furthering versatility in RNA structure and function.[2]. Therefore, RNA modules can be easily incorporated into artificial networks and systems to achieve molecular precision over programmable properties. Nanoscale devices can be designed and programmed to process information in a highly controllable fashion, owing to the predictable behavior of RNA-based elements.

This concept lies at the root of structural RNA nanotechnology and it has been implemented in biomolecular engineering and synthetic biology to generate new molecular functionality [3-5]. Not surprisingly, RNA nanotechnology has also branched out into the field of diagnostics [6], aiming to meet the growing demand for sensing strategies capable of performing sensitive and specific molecular recognition [7]. RNA modules are used to provide a designed system with sequence-specific programmability, which ultimately allows for the development of sensing platforms tailored to desired, single molecule analytes. Nucleic acid biomarkers, such as clinically relevant RNA transcripts (mRNAs) or microRNAs (miRNAs), target proteins, or small molecule metabolites can be imaged and detected in and out of the cellular environment by using rationally designed RNA-based devices. At the boundary of bioanalytical chemistry and synthetic biology, advanced designs can also combine the recognition of molecular targets with on-demand regulation of genetic expression and activation of synthetic gene networks.

In this article, we discuss recent advances in RNA-based sensing platforms and highlight the potential of RNA nanotechnology for biomedical diagnostics. We start by discussing programmable systems that leverage recently developed light-up RNA aptamers to transduce a binding event into a 
readable fluorescence output. In particular, we focus on binding-responsive, dynamic systems in which the activation of a functional RNA reporting unit is the result of either an induced conformational change or the supramolecular assembly of RNA modules. Next, we examine advances in the design of RNA-based switches integrated into synthetic genetic networks that sense specific targets, process information and regulate gene expression through programmed RNA interactions. In this context, we focus on rationally designed toehold switches deployed in living cells for synthetic, target-responsive gene regulation; we then move on and showcase their use as pivotal elements of advanced paper-based platforms for diagnostic applications.

\section{Dynamic sensing devices based on RNA aptamer modules}

Light-up aptamers are a special class of RNA aptamers that turn on the fluorescence emission of small-molecule fluorophores upon binding [8]. Following the early discovery of the fluorescent malachite green RNA aptamer, several light-up aptamers have emerged in the recent years that are able to bind to different biocompatible fluorogenic ligands, paving the way to the design of innovative RNA-based molecular devices for sensing applications [9, 10]. The process underlying the identification of such aptamers is a combinatorial selection procedure called Systematic Evolution of Ligands by EXponential enrichment (SELEX), in which libraries of RNA molecules are subjected to iterative rounds of selection and amplification yielding RNA aptamers with the highest affinity for a desired ligand $[11,12]$. On account of their fluorescence properties, light-up aptamers can be used as versatile reporting units and incorporated into dynamic RNA nanostructures with programmable sensing functionalities. A very elegant and effective way of doing so is to construct RNA-based sensors by fusing a light-up aptamer sequence with a targetresponsive RNA module. In such designs, the aptamer-based reporting unit is initially kept in an off-state by forced, incorrect folding; the interaction with the target molecule then causes a conformational change in the whole RNA structure that leads to correct folding and activation of the fluorescence-upon-binding properties of the light-up aptamer. With regard to this, structure switching RNA-based sensors integrating a binding-responsive malachite green RNA aptamer 
module were initially developed capable of detecting small molecules (adenosine) [13] and clinically relevant target proteins (hepatitis $\mathrm{C}$ helicase and replicase) [14]. More recently, the identification of improved light-up aptamers such as Spinach (including Spinach-derived daughter structures) [15], Broccoli [16], and Mango [17] has given a boost to the development of advanced structure switching RNA-based sensors.

\section{Insert Fig.1}

Jaffrey and coworkers pioneered the use of Spinach to build analyte-responsive RNA nanodevices that function in the cellular environment. In a seminal work, they reported the engineering of a sensor for small molecules which harbors a target-binding RNA aptamer and a Spinach module. This latter binds to a synthetic copy (3,5-difluoro-4-hyroxybenzylidene imidazoline, DFHBI) of the naturally occurring Green Fluorescent Protein (GFP) fluorophore and displays GFP-like fluorescence properties [18]. Binding of the target small molecule to its cognate aptamer promotes the functional folding of the Spinach unit through the stabilization of a shared stem sequence in the sensor ("transducer"), which ultimately results in a readable fluorescence output. Building on this design, several sensors were developed against a variety of target small molecules, including adenosine 5'-diphospate (ADP), S-adenosylmethionine (SAM), and guanosine 5'-triphosphate (GTP), and were further genetically encoded in live cells to monitor metabolite dynamics in real-time [19]. Next, Spinach modules were incorporated into the structure of naturally occurring riboswitches to create fluorescent Spinach riboswitches. These are genetically encoded metabolite sensors that leverage the binding-induced conformational change of the riboswitch structure to trigger the proper folding of the Spinach portion. In this way, an RNA-based sensor for thiamine 5'-pyrophosphate (TPP) was developed that allowed for real-time monitoring of dynamic changes in intracellular TPP concentrations in single cells (Fig. 1A) [20]. As cellular metabolites are known for playing a pivotal role in cell physiology and their altered concentration levels can be associated to disease states [21, 22], the development of innovative sensors capable of performing 
metabolite quantification directly in living cells is likely to push the boundaries of bioanalytics and molecular diagnostics.

Switch-upon-binding RNA structures have also been used as platforms for the detection of target nucleic acid sequences, in particular clinically relevant microRNAs (miRNAs). Aw et al. leveraged the interaction with target miRNAS to zip the nucleotides of a destabilized Spinach-like structure close together, achieving an average 50-fold fluorescence emission enhancement upon miRNA-responsive functional folding of the light-up aptamer portion [23]. Similarly, Huang et al. developed FASTmiR, a miRNA sensor in which the hybridization with a target miRNA, facilitated by the presence of an ad hoc toehold sequence, induces a conformational transition that leads to the formation of a stabilized Spinach structure. FASTmiR sensors against miR-171 and miR-122 were demonstrated able to detect their targets in total RNA extracts within a micromolar range; the expression of a tandem array of FASTmiRs in mammalian cells allowed for fluorescence in situ hybridization of miR-122 [24]. In a recent attempt to improve microRNA imaging in living cells, a genetically encoded structure switching RNA probe was used in tandem with the simultaneous expression of GFP. This was designed to generate a Förster resonance energy transfer (FRET)based ratiometric sensor for imaging and quantification of intracellular miR-21, which is one the of most common microRNA biomarkers aberrantly expressed in many cancers [25]. Here, the hybridization with the target miRNA induces a molecular beacon-like conformational change in the sensor, which, supported by extra-stabilization provided by the fusion with a tRNA scaffold, allows for the correct folding of the light-up aptamer binding to the fluorophore sulforhodamine B (SR). (Fig. 1B) [26]. Based on a similar ratiometric mechanism, the work of Jepsen et al. is a first-time example of constructing aptamer-based FRET systems using single-stranded RNA origami templates. Such RNA nanodevices, which are completely genetically encodable, were obtained by placing a Spinach and a Mango aptamer in close proximity on an RNA scaffold, and were demonstrated to provide a FRET output when conformational changes were induced by hybridization with complementary nucleic acid sequences or by binding to small molecule ligands 
(Fig. 1C) [27]. It is worth noting that Mango, on account of its ability to fluorescently emit in the yellow/orange and near infrared windows upon binding to thiazole orange derivatives, has been also used as genetically encoded RNA tag for imaging of small non-coding RNAs [28].

\section{Insert Fig.2}

Dynamic RNA-based biosensors can alternatively be engineered by using the programmable, target-assisted assembly of functional modules to activate a light-up aptamer reporter. Inspired by protein-based complementation assays [29], light-up aptamers can be split into two halves showing poor hybridization affinity at low concentrations, and a specific biomolecule can serve as a template directing their assembly into the native, functional aptamer structure. Confinement of the aptamer halves within the biomolecule volume translates into a drastic enhancement of their local concentration, which boosts complementary base pairing and assembly into the stabilized RNA structure [30]. In an early example, the malachite green aptamer was used to build a binary RNA-based sensors for target nucleic acids by splitting the original aptamer structure into two independent strands, each tethered to additional binding arms for a specific DNA sequence. In the presence of a complementary DNA to the binding arms, the two RNA fragments were brought in as close proximity as they could hybridize and assemble into the original malachite green aptamer [31]. Kikuchi et al. developed versatile, modular, Spinach-based systems as sensing platforms for target DNA and RNA sequences, achieving limits of detection (LOD) in the nanomolar range. The same systems were also proven to be useful for programmable molecular computation $[32,33]$. Recently, intracellular mRNA transcripts were imaged using a genetically encoded modular RNA sensor. In this case, a Broccoli aptamer was divided in two strands requiring the presence of a target mRNA sequence as a molecular template assisting their assembly into the functional form. Expressing this split architecture in the cell cytoplasm allowed for the real-time imaging of different endogenous mRNAs in both epithelial and stem cells, which suggests this technology might be directed towards any native transcript of interest (Fig. 2A) [34]. Modular, aptamer-based sensors are now also available that respond to protein targets. 
With regard to this, Bertucci, Porchetta, et al. reported the first example of a split-Spinach system designed for the detection of antibodies (Fig. 2B) [35]. Each of two Spinach fragments was conjugated at one end with a ligand (antigen) recognized by a specific antibody. The interaction with the target antibody allowed the two RNA strands to be confined in close proximity and thus to assemble into the functional Spinach structure, which, by binding to the cognate DFHBI ligand, provided a fluorescence signal in an antibody concentration-dependent manner. This system was demonstrated to be efficiently working in complex matrices such as cell culture medium and whole cell lysates, and it could potentially be extended to the detection of any target proteins for which ligands are available that can be conjugated to RNA strands. Modular, aptamer-based sensing devices can be further engineered to feature multiple degrees of molecular motion. For instance, programmable strand displacement reactions can be used to remotely control the assembly of a split Spinach system [36]. Recently, catalytic hairpin assembly (CHA) was combined with a split Broccoli architecture and used to image RNAs of interest in live cells. The Broccoli aptamer was divided in two segments, each of them conjugated to the end of two CHA hairpins, and the interaction with a target intracellular RNA was used to catalytically sustain the hybridization between the two modules and the ensuing formation of the functional aptamer structure (Fig. 2C) [37]. Alam et al. reported the development of a split system based on a dimeric Broccoli architecture stabilized by a three-way RNA junction. This system was initially employed for probing intracellular RNA-RNA interactions, subsequently it was coupled to an RNA regulatory system expressing a reporter fluorescent protein and allowing for monitoring of RNA assembly with multi-fluorescence readout [38]. These last two examples provide also insights into RNA molecular circuitry and synthetic RNA regulation, which will be covered in the following sections.

\section{RNA-based toehold switches: from target-responsive synthetic gene regulation to paper-based diagnostic applications.}

Recent advances in synthetic biology have suggested that biosensor performances may be improved by expanding the range of detectable targets and by developing programmable artificial 
gene networks able to transduce multiple molecular inputs into designed functional outputs [39-41]. Some major limitations remain, though, which have substantially hampered the development of more complex circuits. These include the small number of available genetic circuit components and the difficulty in controlling these elements in an orthogonal fashion [6]. Among the emerging synthetic biology systems, RNA-based devices and networks show a unique ability to sense molecular cues, process the associated information, and direct controllable cell behavior [42-44]. In this sense, engineered RNA-based genetic systems hold the tremendous potential for artificially recreating naturally occurring sensing and regulatory mechanisms.

\section{Insert Fig.3}

The complexity of RNA-based circuits has been drastically improved with the introduction of novel classes of RNA-based regulatory components capable of operating robustly in living cells. Among these, riboregulators were one of the first examples of engineered RNA hairpins working as prokaryotic translational regulators (Fig. 3A) [45, 46]. Their molecular structure prevents the binding of ribosomes to the ribosomal binding site (RBS), thus inhibiting translation initiation. Riboregulators are generally composed of cognate pairs of RNAs: a transducer RNA that regulates either translation or transcription, and a trans-acting sequence that binds to the transducer and tunes its activity. Riboregulators can be classified based on the initial RNA-RNA interaction that triggers the hybridization processes (i.e. loop-loop or loop-linear interactions). Despite the recent progresses in modeling and designing large families of orthogonal RNA riboregulator mutants [47], some major issues still have to be addressed, including modest dynamic ranges (19-fold) and low specificity [48]. Moreover, riboregulator sequences need to share a specific sequence-dependent motif with the RBS to exert their function, which severely limits the extent to which these devices can be useful for diagnostic applications [49].

Green et al. overcame these constraints by introducing a "toehold" domain through which it is possible to control RNA-RNA interactions and efficiently activate translation on-demand. (Fig. 3B) [50]. Specifically, this new design is based on re-engineered RNA hairpins, called "toehold 
switches", exposing only a linear toehold domain that works as a seed region responsible for the hybridization with the input RNA. This architecture benefits from the advantageous thermodynamics and kinetics of linear-linear nucleic acid interactions compared to those of linearloop interactions. Upon binding of the input RNA, the initial hairpin structure unwinds pushed by a toehold-mediated strand displacement reaction; the ensuing exposure of an unconstrained RBS allows for ribosome binding and subsequent translational activity (i.e. protein expression). Importantly, toehold switches are designed to keep the RBS sequestered in the loop of the hairpin, which eliminates the previous need for sequence conservation between the input RNA and the RBS motif. Green et al. developed a box of 13 orthogonal toehold switches that performed detection of different mRNA through the simultaneous monitoring of GFP expression. Such switches also showed a relevant increase in ON/OFF fluorescence ratios (400-fold) comparable or higher than that of protein-based regulators. The integration of these toehold switches into the genome to control translation of endogenous genes also allowed for the simultaneous orthogonal control of 12 genes expression in a single cell. As these last examples suggest, the application of bioengineering principles to RNA-based tools may open up fascinating avenues for the development of nextgeneration molecular diagnostic devices. However, there remain biosafety concerns intrinsically related to the release in the environment of genetically modified organisms that have yet to be properly addressed [51]. Looking forward, future efforts should be directed to translate the RNA switch technology into disposable and user-friendly devices for real-world diagnostic and environmental applications. In order to fulfill this objective, different possible strategies have been recently explored for hosting RNA-based systems outside of the cell environment. In this regard paper-based technologies have been emerging as promising platforms helping translate RNA-based synthetic biology into diagnostic devices.

Paper-based biomedical devices hold the potential to fill the current gap in diagnostic capability between developed and low- and middle-income countries, which, constrained by scarce economical resources, often have to rely on decentralized settings for clinical analysis where low- 
cost and portability of devices are crucial requirements to be met [52]. Recent advances in the field of microfluidics also facilitated the use of paper-based systems as a substrate material for microfluidic assays, thus bringing up many more opportunities for diagnostic research [53]. However, to date very few examples of POC devices capable of reaching the market have been reported. This is mainly due to the fact that scaling down and integrating all the biosensing components in one single device (e.g. chemical storage, detection, transduction, readout, etc.) is still highly challenging. In this context, the combination of paper-based platforms with RNA nanotechnology may help circumvent some of current limitations.

Collins and coworkers pioneered a method to fix synthetic gene networks onto paper and used it to manufacture advanced diagnostic devices [54]. The authors demonstrated that enzymemediated transcription and translation could be reconstituted from freeze-dried, bacterial cell-free systems by simply adding water onto paper. By doing so, they showed that the resulting fluorescence emission of GFP expressed in paper is comparable to the one obtained with standard expression in solution. Furthermore, they demonstrated that both simple cell-free GFP expression and more complex synthetic gene networks are supported while embedded in the cellulose matrix of paper. Indeed, paper-embedded gene networks were stable at room temperature and their activation was triggered by simply adding water to the substrate. Notably, they then combined this paperbased technology with programmable RNA toehold switches in order to develop rapid and costeffective diagnostic sensors against the Ebola virus. More in detail, they identified 24 toehold switches that could distinguish between the Sudan and Zaire strains of the virus at the cost of about $20 \$$ per sensor. The selected toehold switches worked in an orthogonal modality and showed linear response to RNA concentrations, allowing for inferring semi-quantitative information about the input RNA strands. Unfortunately, one of the limitations of such RNA-based biosensing system is its relatively low sensitivity (LOD of the method is $30 \mathrm{nM}$ ), which restricts its applicability for infield applications. 


\section{Insert Fig.4}

Motivated by this proof-of-concept demonstration, Pardee et al. recently improved on the same technology and fabricated paper-based sensors incorporating RNA networks for the detection of the Zika virus RNA genome [55]. In this case, the authors interfaced the biosensing platform with a novel CRISPR/Cas9-based cleavage assay able to discriminate between viral strains with single-base resolution. This paper-based diagnostic platform, allowing for sequence-specific detection of femtomolar concentrations of Zika virus fragments, could be employed in low-resource settings and potentially substitute the standard, laboratory-based, serological assays based on antibody detection, these latter having limited diagnostic fidelity because of the cross-reactivity manifested in patients previously infected with other flaviviruses spread in the same area. Moreover, with the intention of moving towards field-operating diagnostic devices, the authors also tested their paper-based RNA platform using a portable electronic reader, which provided robust and quantitative measurements at a total system cost of about $\$ 250$.

More recently, the same group further explored the potential of this sensing platform for addressing the need for affordable, on-demand, and simple analysis of gut microbiome samples [56] (Fig. 4A). They demonstrated species-specific detection of marker mRNAs from 10 different bacteria with relevance to microbiome research and assessed the capability of quantifying mRNA targets $(\mathrm{LOD}=3 \mathrm{fM})$ using arrays of toehold-switches sensors in paper-based reactions. Detection of mRNAs with femto-molar sensitivity was achieved by harnessing a nucleic acid sequence based amplification step (NASBA), (Fig. 4B) and the method was then validated on stool samples by benchmarking against standard reverse transcription quantitative polymerase chain reaction (RTqPCR) (Fig. 4C). Nevertheless, for the purpose of a large-scale implementation of such synthetic RNA-based systems in various branches of diagnostics, some challenges have yet to be addressed, including limited sensitivity in the absence of amplification steps and complicated sample processing.

\section{Outlook}


The widespread presence of RNA-based regulation mechanisms in natural systems are a great source of inspiration for the design of artificial systems with programmable properties. This, alongside recent progresses in computational tools for RNA design, has channeled growing interest into the field of RNA nanotechnology and synthetic RNA-based biology. In this article, we aimed to report recent trends in the fields of sensing and diagnostics, highlighting how RNA-based modules, recognition elements, and synthetic networks can be manipulated and engineered to perform programmable tasks in and out of the cellular environment. Despite unsolved constraints (although the field is still relatively young), the many examples we have reported herein set forth how the use of engineered RNA-based devices could greatly benefit bioanalytical chemistry by providing novel detection schemes and improved performances in molecular diagnostics and intracellular imaging. As we gain more insights into RNA sequence-structure-function correlation, more sophisticated nature-inspired designs are likely to open up new avenues for the development of next-generation biosensors. We also believe that RNA-based precision diagnostics will flourish as long as interfacing with complementary disciplines is set as a guiding principle. Bringing together RNA programmability with approaches and methodologies borrowed from both neighboring and distant research fields is likely to lay the foundations of a new era of smart biomimetic devices and technologies. Furthermore, we envision that future endeavors aimed at interfacing programmable RNA-based systems with hardware will support the development of realworld sensors useful for environmental analysis, personalized healthcare, and portable electronics.

\section{References}

1. Engelhart AE. RNA imaging: A tale of two G-quadruplexes. Nat Chem Biol. 2017;13(11):1140-1141

2. Guo P. The emerging field of RNA nanotechnology. Nat Nanotechnol. 2010;5(12):833-842.

3. Grabow WW, Jaeger L. RNA self-assembly and RNA nanotechnology. Acc Chem Res. 2014;47(6):1871-1880.

4. Jasinski D, Haque F, Binzel DW, Guo P. Advancement of the Emerging Field of RNA 
Nanotechnology. ACS Nano. 2017;11(2):1142-1164.

5. Chappell J, Watters KE, Takahashi MK, Lucks JB. A renaissance in RNA synthetic biology: New mechanisms, applications and tools for the future. Curr Opin Chem Biol. 2015;28:4756.

6. Slomovic S, Pardee K, Collins JJ. Synthetic biology devices for in vitro and in vivo diagnostics. Proc Natl Acad Sci U S A. 2015;112(47):14429-14435 .

7. Bailey RC. Grand Challenge Commentary: Informative diagnostics for personalized medicine. Nat Chem Biol. 2010;6(12):857-859 .

8. Bouhedda F, Autour A, Ryckelynck M. Light-up RNA aptamers and their cognate fluorogens: From their development to their applications. Int J Mol Sci. 2018;19(1):E44 .

9. Neubacher S, Hennig S. RNA Structure and Cellular Applications of Fluorescent Light-Up Aptamers. Angew Chemie Int Ed. 2018; doi: 10.1002/anie.201806482

10. Ouellet J. RNA Fluorescence with Light-Up Aptamers. Front Chem. 2016;4:29.

11. Szeto K, Latulippe DR, Ozer A, Pagano JM, White BS, Shalloway D, Lis JT, Craighead HG. RAPID-SELEX for RNA aptamers. PLoS One. 2013;8(12):e82667

12. Gotrik M, Sekhon G, Saurabh S, Nakamoto M, Eisenstein M, Soh HT. Direct Selection of Fluorescence-Enhancing RNA Aptamers. J Am Chem Soc. 2018;140(10):3583-3591.

13. $\mathrm{Xu} \mathrm{W,} \mathrm{Lu} \mathrm{Y.} \mathrm{Label-Free} \mathrm{Fluorescent} \mathrm{Aptamer} \mathrm{Sensor} \mathrm{Based} \mathrm{on} \mathrm{Regulation} \mathrm{of} \mathrm{Malachite}$ Green Fluorescence. Anal Chem. 2010;82(2):574-578.

14. Bang GS, Cho S, Lee N, Lee B-R, Kim J-H, Kim B-G. Rational design of modular allosteric aptamer sensor for label-free protein detection. Biosens Bioelectron. 2013;39(1):44-50.

15. Paige JS, Wu KY, Jaffrey SR. RNA Mimics of Green Fluorescent Protein. Science. 2011; 333(6042):642-646.

16. Filonov GS, Moon JD, Svensen N, Jaffrey SR. Broccoli: Rapid Selection of an RNA Mimic of Green Fluorescent Protein by Fluorescence-Based Selection and Directed Evolution. J Am Chem Soc. 201;136(46):16299-16308. 
17. Dolgosheina E V., Jeng SCY, Panchapakesan SSS, Cojocaru R, Chen PSK, Wilson PD, Hawkins N, Wiggins PA, Unrau PJ. RNA Mango Aptamer-Fluorophore: A Bright, HighAffinity Complex for RNA Labeling and Tracking. ACS Chem Biol. 2014;9(10):2412-2420.

18. Warner KD, Chen MC, Song W, Strack RL, Thorn A, Jaffrey SR, Ferré-D’Amaré AR. Structural basis for activity of highly efficient RNA mimics of green fluorescent protein. Nat Struct Mol Biol. 2014;21(8):658-663.

19. Paige JS, Nguyen-Duc T, Song W, Jaffrey SR. Fluorescence Imaging of Cellular Metabolites with RNA. Science. 2012;335(6073):1194.

20. You M, Litke JL, Jaffrey SR. Imaging metabolite dynamics in living cells using a Spinachbased riboswitch. Proc Natl Acad Sci. 2015;112(21):E2756

21. Park MH, Igarashi K. Polyamines and their metabolites as diagnostic markers of human diseases. Biomol Ther. 2013;21(1):1-9.

22. Fernie AR, Trethewey RN, Krotzky AJ, Willmitzer L. Metabolite profiling: from diagnostics to systems biology. Nat Rev Mol Cell Biol. 2004;5(9):763-769.

23. Aw SS, Tang MX, Teo YN, Cohen SM. A conformation-induced fluorescence method for microRNA detection. Nucleic Acids Res. 2016;44(10):e92

24. Huang K, Doyle F, Wurz ZE, Tenenbaum SA, Hammond RK, Caplan JL, Meyers BC. FASTmiR: an RNA-based sensor for in vitro quantification and live-cell localization of small RNAs. Nucleic Acids Res. 2017;45(14):e130

25. Krichevsky AM, Gabriely G. miR-21: a small multi-faceted RNA. J Cell Mol Med. 2008;13(1):39-53.

26. Ying Z-M, Wu Z, Tu B, Tan W, Jiang J-H. Genetically Encoded Fluorescent RNA Sensor for Ratiometric Imaging of MicroRNA in Living Tumor Cells. J Am Chem Soc. 2017;139(29):9779-9782.

27. Jepsen MDE, Sparvath SM, Nielsen TB, Langvad AH, Grossi G, Gothelf K V, Andersen ES. Development of a genetically encodable FRET system using fluorescent RNA aptamers. Nat 
Commun. 2018;9(1):18.

28. Autour A, C Y Jeng S, D Cawte A, Abdolahzadeh A, Galli A, Panchapakesan SSS, Rueda D, Ryckelynck M, Unrau PJ. Fluorogenic RNA Mango aptamers for imaging small non-coding RNAs in mammalian cells. Nat Commun. 2018;9(1):656.

29. Michnick SW, Ear PH, Manderson EN, Remy I, Stefan E. Universal strategies in research and drug discovery based on protein-fragment complementation assays. Nat Rev Drug Discov. 2007;6(7):569-582.

30. Zhang H, Li F, Dever B, Wang C, Li X-F, Le XC. Assembling DNA through Affinity Binding to Achieve Ultrasensitive Protein Detection. Angew Chemie Int Ed. 2013;52(41):10698-10705.

31. Kolpashchikov DM. Binary Malachite Green Aptamer for Fluorescent Detection of Nucleic Acids. J Am Chem Soc. 2005;127(36):12442-12443.

32. Kikuchi N, Kolpashchikov DM. Split Spinach Aptamer for Highly Selective Recognition of DNA and RNA at Ambient Temperatures. ChemBioChem. 2016;17(17):1589-1592.

33. Kikuchi N, Kolpashchikov DM. A universal split spinach aptamer (USSA) for nucleic acid analysis and DNA computation. Chem Commun. 2017;53(36):4977-4980.

34. Wang Z, Luo Y, Xie X, Hu X, Song H, Zhao Y, Shi J, Wang L, Glinsky G, Chen N, Lal R, Fan C. In Situ Spatial Complementation of Aptamer-Mediated Recognition Enables LiveCell Imaging of Native RNA Transcripts in Real Time. Angew Chemie Int Ed 2018;57(4):972-976.

35. Bertucci A, Porchetta A, Ricci F. Antibody-Templated Assembly of an RNA Mimic of Green Fluorescent Protein. Anal Chem. 2018;90(2):1049-1053.

36. Rogers TA, Andrews GE, Jaeger L, Grabow WW. Fluorescent monitoring of RNA assembly and processing using the split-spinach aptamer. ACS Synth Biol. 2015;4(2):162-166.

37. Karunanayake Mudiyanselage APKK, Yu Q, Leon-Duque MA, Zhao B, Wu R, You M. Genetically Encoded Catalytic Hairpin Assembly for Sensitive RNA Imaging in Live Cells. J 
Am Chem Soc. 2018;140(28):8739-8745.

38. Alam KK, Tawiah KD, Lichte MF, Porciani D, Burke DH. A Fluorescent Split Aptamer for Visualizing RNA-RNA Assembly in Vivo. ACS Synth Biol. 2017;6(9):1710-1721.

39. Xie M, Fussenegger M. Designing cell function: assembly of synthetic gene circuits for cell biology applications. Nat Rev Mol Cell Biol. 2018;19(8):507-525.

40. Masubuchi T, Endo M, Iizuka R, Iguchi A, Yoon DH, Sekiguchi T, Qi H, Iinuma R, Miyazono Y, Shoji S, Funatsu T, Sugiyama H, Harada Y, Ueda T, Tadakuma H. Construction of integrated gene logic-chip. Nat Nanotechnol. 2018;13(10):933-940.

41. Nielsen AAK, Der BS, Shin J, Vaidyanathan P, Paralanov V, Strychalski EA, Ross D, Densmore D, Voigt CA. Genetic circuit design automation. Science. 2016;352(6281):7341

42. McKeague M, Wong RS, Smolke CD. Opportunities in the design and application of RNA for gene expression control. Nucleic Acids Res. 2016;44(7):2987-2999.

43. Etzel M, Mörl M. Synthetic Riboswitches: From Plug and Pray toward Plug and Play. Biochemistry. 2017;56(9):1181-1198.

44. Chen YY, Jensen MC, Smolke CD. Genetic control of mammalian T-cell proliferation with synthetic RNA regulatory systems. Proc Natl Acad Sci U S A. 2010;107(19):8531-8536.

45. Isaacs FJ, Dwyer DJ, Ding C, Pervouchine DD, Cantor CR, Collins JJ. Engineered riboregulators enable post-transcriptional control of gene expression. Nat Biotechnol. 2004:22(7):841-847.

46. Bayer TS, Smolke CD. Programmable ligand-controlled riboregulators of eukaryotic gene expression. Nat Biotechnol. 2005;23(3):337-343.

47. Mutalik VK, Qi L, Guimaraes JC, Lucks JB, Arkin AP. Rationally designed families of orthogonal RNA regulators of translation. Nat Chem Biol. 2012;8(5):447-454.

48. Liu CC, Qi L, Lucks JB, Segall-Shapiro TH, Wang D, Mutalik VK, Arkin AP. An adaptor from translational to transcriptional control enables predictable assembly of complex regulation. Nat Methods. 2012;9(11):1088-1094. 
49. Isaacs FJ. Synthetic biology: Automated design of RNA devices. Nat Chem Biol. 2012;8(5):413-415.

50. Green AA, Silver PA, Collins JJ, Yin P. Toehold switches: De-novo-designed regulators of gene expression. Cell. 2014;159(4):925-939.

51. Karig DK. Cell-free synthetic biology for environmental sensing and remediation. Curr Opin Biotechnol. 2017;45:69-75.

52. Parolo C, Merkoçi A. Paper-based nanobiosensors for diagnostics. Chem Soc Rev. 2013;42:450-457.

53. Yamada K, Shibata H, Suzuki K, Citterio D. Toward practical application of paper-based microfluidics for medical diagnostics: state-of-the-art and challenges. Lab Chip 2017;17:1206-1249.

54. Pardee K, Green AA, Ferrante T, Cameron DE, Daleykeyser A, Yin P, Collins JJ. Paperbased synthetic gene networks. Cell. 201;159(4):940-954.

55. Pardee K, Green AA, Takahashi MK, Braff D, Lambert G, Lee JW, Ferrante T, Ma D, Donghia N, Fan M, Daringer NM, Bosch I, Dudley DM, O’Connor DH, Gehrke L, Collins JJ. Rapid, Low-Cost Detection of Zika Virus Using Programmable Biomolecular Components. Cell. 2016;165(5):1255-1266.

56. Takahashi MK, Tan X, Dy AJ, Braff D, Akana RT, Furuta Y, Donghia N, Ananthakrishnan A, Collins JJ. A low-cost paper-based synthetic biology platform for analyzing gut microbiota and host biomarkers. Nat Commun. 2018;9:3347. 
a

Minimal Fluorescence Strong Fluorescence

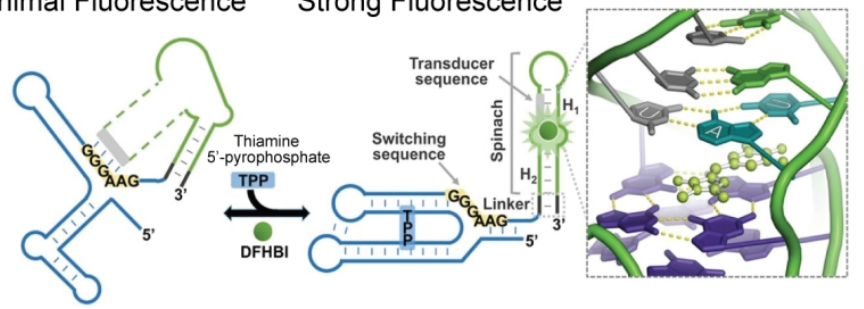

b

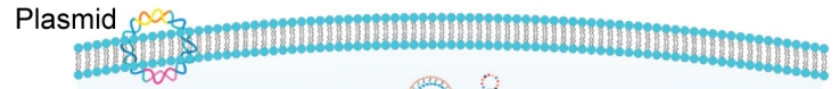

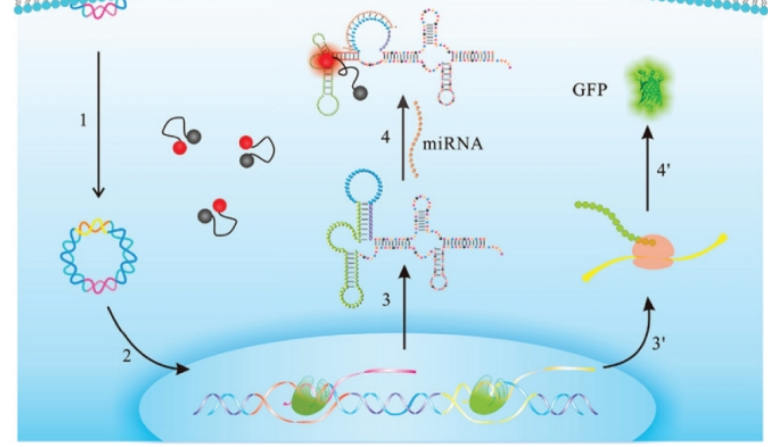

C
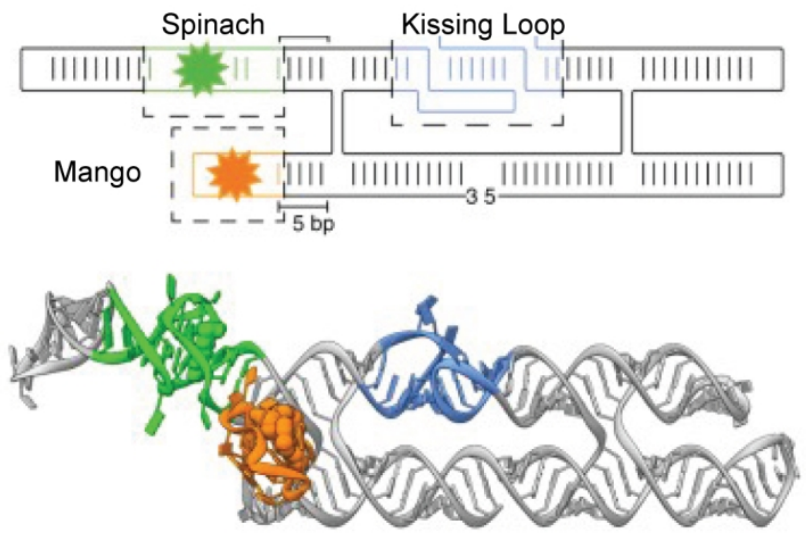

$101 \times 172 \mathrm{~mm}(300 \times 300$ DPI $)$ 
a

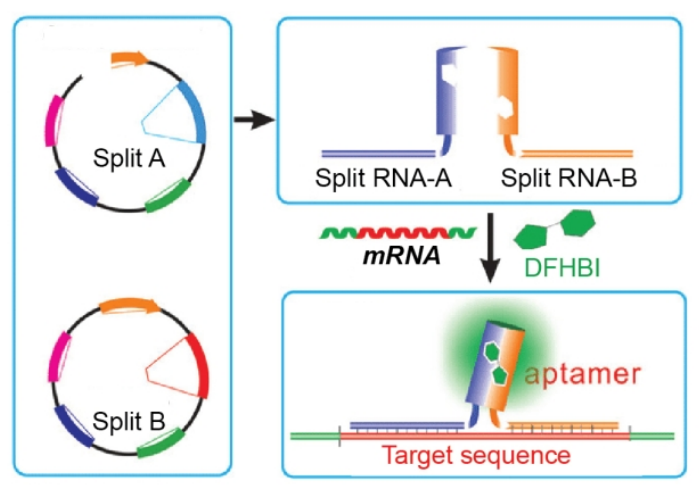

b

Split Spinach aptamer
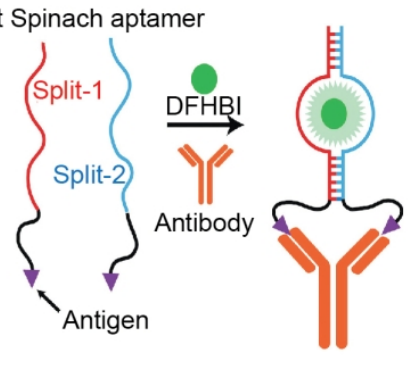

DFHBI Split $1 \quad A b$

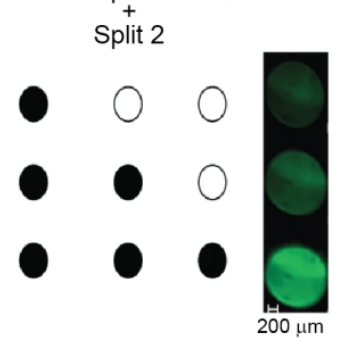

C

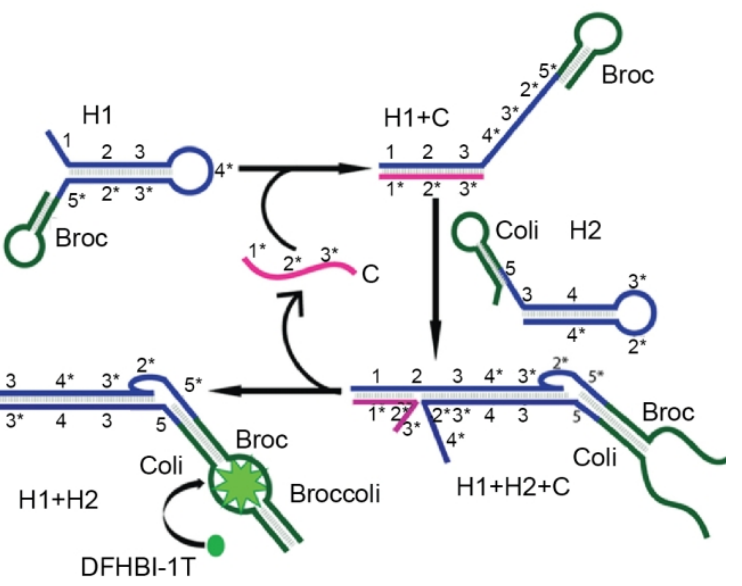


a Riboregulator switches (Translation)

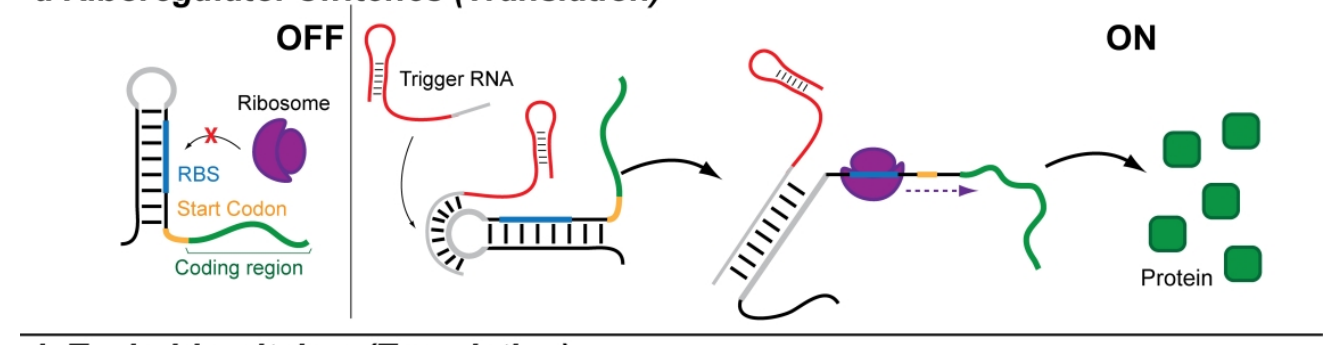

b Toehold switches (Translation)
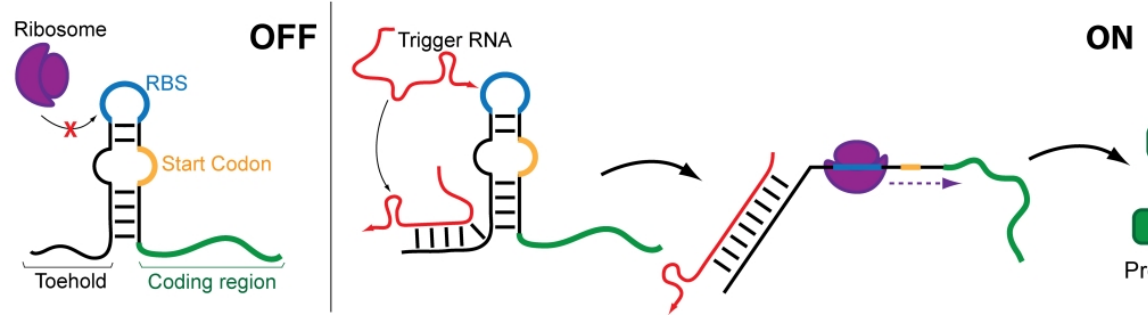

$399 \times 223 \mathrm{~mm}(300 \times 300$ DPI $)$ 


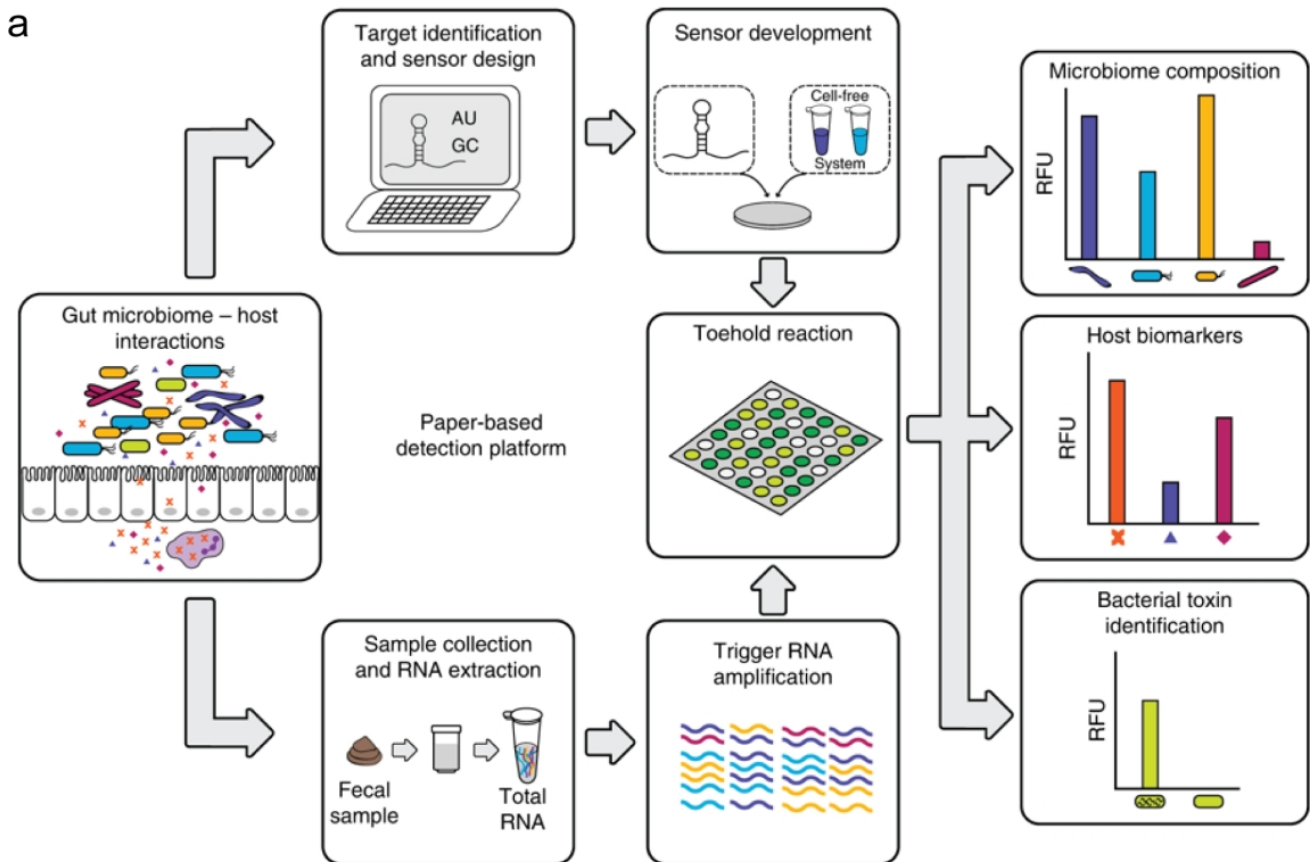

b

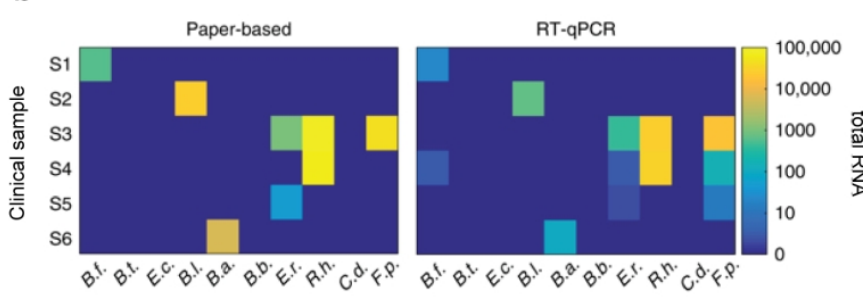

$160 \times 160 \mathrm{~mm}(300 \times 300$ DPI $)$
C

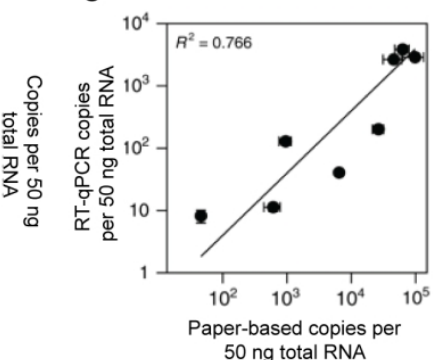

$50 \mathrm{ng}$ total RNA 


\section{Figure captions}

Fig. 1 Aptamer-based dynamic sensing devices with their functionality controlled by bindingresponsive conformational changes. (a) A riboswitch is coupled with a reporter Spinach aptamer to image cellular metabolites, i.e. TPP, in living cells. the Spinach aptamer (transducer domain) hybridizes with the TPP-binding switching portion thus inhibiting the DFHBI binding. In the presence of TPP, the system undergoes a conformational change that brings the Spinach aptamer in its active state allowing DFHBI binding and consequent fluorescence emission. Adapted with permission from Proceedings of the National Academy of Sciences USA You M, Litke JL, Jaffrey SR. J. Proc. Natl. Acad. Sci. U. S. A. 2015, 112, E2756-E2765. (b) The sulforhodamine B-binding aptamer is fused with a miR-responsive stem-loop motif to image microRNA in living tumor cells. The binding of the target miRNA to the stem-loop structure induces a conformation change resulting in the activation of the binding conformation of the sulforhodamine B-binding aptamer. The simultaneous co-expression of the RNA sensor with GFP allows for ratiometric and quantitative detection of target miRNAs in living cells. Adapted with permission from Ying Z-M, Wu Z, Tu B, Tan W, Jiang J-H. Genetically Encoded Fluorescent RNA Sensor for Ratiometric Imaging of MicroRNA in Living Tumor Cells. J Am Chem Soc 139:9779-9782. Copyright 2017 American Chemical Society. (c) A genetically encodable apta-FRET system structured upon an RNA origami is engineered to respond to the presence of nucleic acid sequences or small molecules in cells. Two fluorescent Mango and Spinach aptamers are placed in close proximity in a singlestranded RNA origami and a kissing-loop portion is inserted into the structure to provide extrasensitivity to conformational changes. Sensing of the targets is achieved by monitoring the change in FRET values upon switching of the biosensor structure. Adapted with permission from Macmillan Publishers Ltd: Nature Communications. Jepsen MDE, Sparvath SM, Nielsen TB, Langvad AH, Grossi G, Gothelf K V, Andersen ES (2018) Development of a genetically encodable FRET system using fluorescent RNA aptamers. Nat Commun 2018, 9, 18. Copyright 2018.

Fig. 2 Binding-responsive sensing devices based on split light-up RNA aptamer. (a) A split Broccoli aptamer is used for imaging of intracellular mRNA transcripts. The co-localization of the two split portions (split A and split B) on the same mRNA target-sequence supports their assembly into the functional aptamer form that allows the DFHBI binding. Adapted from In Situ Spatial Complementation of Aptamer-Mediated Recognition Enables Live-Cell Imaging of Native RNA Transcripts in Real Time. Wang, Z.; Luo, Y.; Xie, X.; Hu, X.; Song, H.; Zhao, Y.; Shi, J.; Wang, L.; Glinsky, G.; Chen, N.; Lal, R.; Fan, C. Angew. Chem. Int. Ed. Engl., Vol. 57, Issue 44. Copyright 2018 Wiley. (b) A modular split Spinach-based platform is developed for the detection 
of antibodies. The two fragments of a split Spinach are both terminally conjugated with an antigen recognized by a specific antibody. Upon interaction with the target antibody, the two antigen-tagged RNA strands are brought in close proximity and can efficiently assemble into the native Spinach structure. Fluorescence microscopy analysis demonstrates that green fluorescence emission is achieved only in the presence of the specific antibody. Adapted from Bertucci, A.; Porchetta, A.; Ricci, F. Anal. Chem. 2018, 90, 1049-1053. Copyright 2018 American Chemical Society. (c) A split Broccoli aptamer is combined with catalytic hairpin assembly (CHA) reaction for imaging of RNAs of interest in living cells. The two portions of a split Broccoli aptamer ("Broc" and "Coli”) are fused with two CHA hairpins ( $\mathrm{H} 1$ and $\mathrm{H} 2)$. The interaction with a specific target RNA (C strand) catalytically activates the hybridization mechanism and, consequently, the folding of the fluorogenic conformation of the aptamer. Adapted from Karunanayake Mudiyanselage, A. P. K. K.; Yu, Q.; Leon-Duque, M.A.; Zhao, B.; Wu, R.; You, M. J. Am. Chem. Soc. 2018, 140, 8739-8745. Copyright 2018 American Chemical Society.

Fig. 3 Synthetic RNA-based regulators of gene expression. (a) Riboregulators are RNA-based stem-loop structures able to inhibit translation in cis by sequestering the RBS of a gene. The binding of a trans-activating RNA triggers the translation. (b) Toehold switches re-engineer riboregulators of gene expression by sequestering the RBS of a gene in the loop of the hairpin. The binding of trigger RNAs to the toehold portion of the switch activate the unzips of the hairpin and the translation. This design prevents ribosome binding and exploits the kinetic and thermodynamic advantages of linear-linear RNA interactions.

Fig. 4 Paper-Based synthetic RNA switches for diagnostic applications. (a) Workflow of a paper-based diagnostic platform for the detection and quantification of bacterial and host RNAs in stool samples exploiting toehold switches. By using commercially available kits, total RNA is extracted from human fecal samples and specific RNAs are amplified via nucleic acid sequence based amplification (NASBA). Paper-based arrays of toehold switch sensors allows for the quantification of the microbial and host biomarker RNA concentration using simple calibration curves. (b) Validation of the paper-based diagnostic platform by comparison with standard RTqPCR analysis of six clinical stool samples. Both the methods identified six bacteria and (c) a good correlation of clinical samples results is obtained with an $\mathrm{R}^{2}$ value of 0.766 . Reprinted by permission from Macmillan Publishers Ltd: NATURE COMMUNICATION, Takahashi, M.K.; Tan, X.; Dy, A.J.; Braff, D.; Akana, R.T.; Furuta, Y.; Donghia, N.; Ananthakrishnan, A.; Collins, J.J., Nat. Commun. 2018, 9, 3347. 